\title{
The Effect Of Training Frequency On Selected Physical And Biochemical Health Parameters In The Conditioning And Reconditioning Of Sedentary Employees
}

\author{
Marlene C. Opperman, University of the Free State, South Africa
} Gert L. Strydom, North-West University, South Africa

\begin{abstract}
Non-communicable disease evolved as a major health risk in South Africa and accounts for about $37 \%$ of all deaths. This burden also challenges human resources in the corporate environment where poor health status (high health risks) may lead to increased health care costs, lowered productivity and eventually to premature death. Some of the South African companies have tackled this challenge by providing various employee health promotion initiatives. One of the popular contributions in this endeavour is to encourage employees to become more physically active in order to prevent detrimental health risk factors or to remedy the existing factors. A major challenge in this initiative is to keep up the compliance of the employees with their physical conditioning programmes, especially when business responsibilities take them away from their health facilities which, in turn, may lead to physical deconditioning. This endorses the motivation of this research as little information is available in South Africa on the aspect of conditioning, deconditioning and reconditioning in the corporate environment.
\end{abstract}

In this study, 60 healthy but sedentary employees, ages 28-49 years, from an academic institution were recruited to participate. They were randomly assigned to three groups, where Groups A and $B$ form the experimental groups with Group $C$ the control group. During the initial training phase (first 12 weeks) Group A and B followed a training frequency of three times per week, while retraining took place at a frequency of two and four times per week for Groups $A$ and $B$, respectively. Group $C$ was not involved in any intervention and continued with their normal daily activities and lifestyle. Results of this study indicated that after training at a program frequency of three times per week, a salutogenic response occurred in the selected physical and biochemical health parameters. With deconditioning, about 50\% of the physical benefits were lost, which occurred faster than the decline in the biochemical (lipids) benefits. With reconditioning, a program frequency of two times per week seems to maintain the physiological status, while with a program frequency of four times per week, an improvement reoccurred, exceeding the benefits of the program frequency of three times per week. In conclusion, it is clear that a physical intervention regime, to the apparently healthy employees, can decrease some health risks; but with an interruption of the program, salutogenic benefits will be partially lost. With retraining, a program frequency of two times per week will not produce significant improvement, but will only maintain the physical status.

Keywords: Program Frequency; Training; Retraining; Physical Fitness; Lipids; Conditioning 


\section{INTRODUCTION}

t has been well established that the health and well-being of human resources in the corporate environment is a crucial factor, by not only determining the productivity of the company, but also by maintaining and improving the personal health of employees (Edington, 2006; Grace, Wilders, Strydom $\&$ Ellis, 2012). This has led to the provision of various health promotion initiatives in corporate settings worldwide (World Economic Forum, 2013). In this respect, Stokes, Henley and Herget (2006) indicate that wellness programmes in the workplace have great potential on employee's health as they spend the major part of their time at the work place (The World Economic Forum, 2009).

The Global Risk Landscape Assessment Report identifies non-communicable (NCD) and chronic diseases as one of the most significant threats facing global economics (IFPMA, 2011). This epidemic of NCD not only forms one of the main drivers of health care costs, but also impacts negatively on productivity (Kolbe-Alexander, Buckmaster, Nossel, Dreyer, Bull, Noakes \& Lambert, 2008). This trend is also evident in South Africa, where 37\% of all deaths are due to NCD (Kolbe-Alexander et al., 2008; World Economic Forum, 2009), leading to the fact that various companies in South Africa now invest in a variety of wellness programmes for their employees (Labuschagne, 2006; Kalas, Kirsten, Strydom \& Wilders, 2012).

The significant role that physical activity can play in combating the epidemic of NCD is already well established (Lambert \& Kolbe-Alexander, 2006) as some researchers indicated that physical activity promises to be one of the most effective intervention strategies, reducing the risks of virtually all chronic diseases simultaneously (Booth, Gordon, Carlson \& Hamilton, 2000). In order to establish the desired outcome of a physical training intervention, various program determinants viz. frequency, intensity, time and type of exercise should be balanced the so-called FITT approach (Oberg, 2007; Opperman \& Strydom, 2012). In this respect, Booth et al. (2000) indicate that participants who exercise to "sweat" at a frequency of one time per week were $24 \%$ less likely to develop type 2 diabetes, compared to those who did not exercise. When the frequency increases to 2-4 times per week, the incidence of type 2 diabetes was reduced by $39 \%$ (Booth et al., 2000).

Various research reports that indicate the prevalence of health risk factors related to NCD in the South African corporate environment are available (Kolbe-Alexander et al., 2008) and often forms the focus point of intervention initiatives (Grace et al., 2012). In this respect, Edington (2001) states that in contrast with previous approaches where high health risk individuals ( $>5$ risk factors) were targeted for intervention, the low-risk individuals $(<2$ risk factors) should not be neglected (Edington, 2001). One of the reasons for this is that employee's health status is not static, and that $2-4 \%$ of the low risk individuals may annually migrate to a higher health risk category, if intervention strategies are not in place to keep them in the low risk category (Musich, McDonald, Hirschland \& Edington, 2003).

The experience of health professionals involved in wellness programmes are that participants often have to interrupt their exercise training regimen due to work-related or other matters (Opperman \& Strydom, 2012). Various questions are then posed as to the effect of detraining and retraining and/or the frequency impact during the retraining phase. Research in this regard is not readily available; therefore, the aim of this study was to determine the effect of training frequency during the training and retraining phases of a conditioning program on selected physical and biochemical health parameters of apparently healthy employees in a South African institution of higher learning. Secondly, the magnitude of detraining and retraining after an initial period of training will also be investigated.

\section{MATERIALS AND METHODS}

\subsection{Study Design}

The design of this research was based on a pretest - post-test protocol with a randomized selection of the experimental and control groups (Thomas, Nelson \& Silverman, 2011). 


\subsection{Participants}

Sixty (60) apparently healthy, but sedentary, Caucasian males between the ages of 28 and 49 years were recruited to participate in this study. None of them took any medication prior to or during the study and all of them were employed in an academic environment. Participants were randomly divided into three groups of 20 each. Group A and B formed the experimental groups who participated in a training - and retraining regimen based on various frequencies, while Group $\mathrm{C}$ acted as a control group and proceeded with their normal lifestyle. Due to the extended period (36 weeks) of this study, a considerable dropout figure in all groups was experienced, primarily caused by moving out of town, lack of motivation and illness. At the end of this study, data of only 13 participants of Group A, 16 of Group B, and 9 of Group C could be analysed.

\subsection{Training, Detraining and Retraining}

For the first 12 weeks the experimental groups (A \& B) trained at a frequency of three times per week, starting at an intensity of $60 \%$ of each individual's age-related maximal heart rate (MHR), determined by the formula of Karvonen (ACSM, 2010). In order to ensure progression, the intensity was increased every two weeks by $5 \%$, causing the participants to reach $85 \%$ of their MHR (ACSM, 2010) by the end of the 12 weeks of training. After a warming-up of five minutes, aerobic training was performed on Monark bicycle ergometers (Model 864) for 30 minutes continuously. This was followed by flexibility and muscle strengthening exercises, using resistance equipment. The flexibility training primarily focused on low-back, shoulder and legs, while the muscle strengthening was directed to the upper body and abdominal muscles. The total duration of each session was 50-60 minutes.

After the 12 weeks of training, a period of detraining (12 weeks) followed, where participants did not engage in any form of training, except their normal daily duties. In the retraining phase (12 weeks) the same exercise training principles applied as in the training phase, except that Group A retrained at a frequency of two times per week, while Group B retrained at a frequency of four times per week. Assessments were conducted at the following intervals: Test 1 - baseline data, Test 2 - after 12 weeks of training; Test 3 -after 12 weeks of detraining; and Test 4 - after 12 weeks of retraining.

\subsection{Physical Working Capacity (PWC) (Watt)}

The PWC of participants was determined by using a multistage bicycle ergometer test, performed on a Monark bicycle ergometer (Model 864). After adjusting the height of the saddle and allowing 2-3 minutes pedaling for warming-up, the initial workload was started at 75 watts. Every five minutes the load was increased by 25 watt up to the stage where the individual reached his predetermined target heart rate of $85 \%$, using the formula of Karvonen (ACSM, 2010) or requested to stop due to fatigue. The workload, when reaching this level, was indicated as the absolute peak physical working capacity (PWC-peak) (watt). The physical capacity (relative value) was then determined by the following PWC (Rel.) $=$ Peak watt $\div$ Body mass $(\mathrm{kg})($ Opperman $\&$ Strydom, 2012).

\subsection{Body Mass (kg)}

The body mass was determined to the nearest $0.1 \mathrm{~kg}$ using a Detecto scale. Participants were only allowed to wear an exercise short.

\subsection{Biochemical Analysis}

A blood specimen was collected from each participant from the antecubital vein following a fasting period of 10 hours. Biochemical analyses were performed by the pathology laboratory of the South African Institute for Medical Research by using the following methods: Total cholesterol - CHOD - PAP method (Richmond, 1973), HDL-C (Burstein, Scholnick \& Morfin, 1970), LDL-C (Friedewald, Levy \& Friederickson, 1972), Triglyceride GPO - (Cole, Klotzsch \& Namara, 1997), TC/HDL -ratio (McArdle, Katch \& Katch, 1996). 


\subsection{Statistical Analysis}

Statistical analysis of this study was done by the Centre for Statistics at the University of the Free State, by using the SPSS software programme. For the evaluation of intragroup differences, the paired t-test was used while, for the intergroup differences, the one-way variance analysis (ANOVA) for multiple dependant variables was used. The Scheffe's test was used to determine statistical significant differences $(\mathrm{p} \leq 0.05)$ between the groups.

\section{RESULTS}

The results of this study are presented in Tables 1 and 2 as well as Figures 1 and 2. The magnitude of change in each of the parameters is presented as the percentage $(\%)$ of change calculated from the baseline values $\left(\mathrm{T}_{1}\right)$. It must be kept in mind that in Table 2, a negative magnitude (\%) represents an improvement in that specific parameter and is therefore reflected as an improvement in Figure 2 and Figure 2 Continue, except for HDL, where a negative value indicates deterioration. 
Table 1: The Effect of Training Frequency on Some Selected Physical Health Parameters in the Training and Retraining of Sedentary Male Employees 1. Physical Working Capacity - Absolute [Peakload in Watt]

\begin{tabular}{|c|c|c|c|c|c|c|c|c|c|c|c|c|c|c|c|c|c|c|c|}
\hline \multirow[t]{3}{*}{ 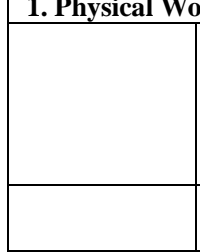 } & \multirow{2}{*}{\multicolumn{4}{|c|}{$\begin{array}{c}\text { Test } 1 \\
\text { Before Training }\end{array}$}} & \multirow{2}{*}{\multicolumn{3}{|c|}{$\begin{array}{c}\text { Test } 2 \\
\text { After 12 Weeks of } \\
\text { Training: 3x/Week }\end{array}$}} & \multirow{2}{*}{\multicolumn{3}{|c|}{$\begin{array}{c}\text { Test } 3 \\
\text { After } 12 \text { Weeks of } \\
\text { Detraining }\end{array}$}} & & & & & & & & & \\
\hline & & & & & & & & & & & \multicolumn{3}{|c|}{$\begin{array}{c}\text { Test } 4 \\
\text { After } 12 \text { Weeks of } \\
\text { Retraining } \\
(\mathbf{A}=2 \mathrm{x} / \text { Week; } \\
\mathbf{B}=4 \mathrm{x} / \text { Week })\end{array}$} & \multicolumn{6}{|c|}{ Intragroup Differences } \\
\hline & & $\overline{\mathbf{x}}$ & SD & $\begin{array}{l}\text { Min } \\
\text { Max }\end{array}$ & $\overline{\mathbf{x}}$ & SD & $\begin{array}{l}\text { Min } \\
\text { Max }\end{array}$ & $\overline{\mathbf{x}}$ & SD & $\begin{array}{l}\text { Min } \\
\text { Max }\end{array}$ & $\overline{\mathbf{X}}$ & SD & $\begin{array}{l}\text { Min } \\
\text { Max }\end{array}$ & $1-2$ & $1-3$ & $1-4$ & $2-3$ & $2-4$ & 3-4 \\
\hline \multirow{3}{*}{ Groups } & $\begin{array}{c}\mathrm{A}(\mathrm{n}=13) \\
\Delta \%\end{array}$ & 150 & 38.2 & $\begin{array}{c}125.0 \\
250\end{array}$ & $\begin{array}{l}182.7 \\
21.8 \%\end{array}$ & 35.9 & $\begin{array}{l}125 \\
250\end{array}$ & $\begin{array}{c}169.2 \\
12.8 \%\end{array}$ & 32.5 & $\begin{array}{l}125 \\
250\end{array}$ & $\begin{array}{c}169.2 \\
12.8 \%\end{array}$ & 32.5 & $\begin{array}{l}125 \\
250\end{array}$ & $\begin{array}{c}* \\
0.01\end{array}$ & $\begin{array}{c}* \\
0.01\end{array}$ & $\begin{array}{c}* \\
0.01\end{array}$ & $\begin{array}{c}* \\
0.01\end{array}$ & $\begin{array}{c}* \\
0.01\end{array}$ & $\begin{array}{c}* \\
1.0\end{array}$ \\
\hline & $\begin{array}{c}\mathrm{B}(\mathrm{n}=16) \\
\Delta \%\end{array}$ & 160.9 & 24.1 & $\begin{array}{l}125 \\
250 \\
\end{array}$ & $\begin{array}{l}189.1 \\
17.5 \% \\
\end{array}$ & 31.6 & $\begin{array}{l}125 \\
250 \\
\end{array}$ & $\begin{array}{l}176.6 \\
9.8 \% \\
\end{array}$ & 29.5 & $\begin{array}{l}125 \\
225 \\
\end{array}$ & $\begin{array}{c}198.4 \\
23.3 \% \\
\end{array}$ & 29.5 & $\begin{array}{l}150 \\
250 \\
\end{array}$ & $\begin{array}{c}* \\
0.01 \\
\end{array}$ & $\begin{array}{c}* \\
0.02 \\
\end{array}$ & $\begin{array}{c}* \\
0.01 \\
\end{array}$ & $\begin{array}{c}* \\
0.02 \\
\end{array}$ & $\begin{array}{c}* \\
0.03 \\
\end{array}$ & $\begin{array}{c}* \\
0.01 \\
\end{array}$ \\
\hline & $\begin{array}{c}\mathrm{C}(\mathrm{n}=9) \\
\Delta \%\end{array}$ & 138.9 & 28.3 & $\begin{array}{l}100 \\
200\end{array}$ & $\begin{array}{c}144.4 \\
4 \%\end{array}$ & 27.3 & $\begin{array}{l}100 \\
200\end{array}$ & $\begin{array}{c}147.2 \\
6 \%\end{array}$ & 23.2 & $\begin{array}{l}125 \\
200\end{array}$ & $\begin{array}{l}150 \\
8 \%\end{array}$ & 21.7 & $\begin{array}{l}125 \\
200\end{array}$ & 0.35 & 0.08 & $\begin{array}{c}* \\
0.04\end{array}$ & 0.59 & 0.35 & 0.35 \\
\hline \multirow{3}{*}{$\begin{array}{l}\text { Intergroup } \\
\text { Differences }\end{array}$} & A vs B & NB & & & NB & & & NB & & & $*$ & & & & & & & & \\
\hline & A vs $\mathrm{C}$ & NB & & & $*$ & & & NB & & & NB & & & & & & & & \\
\hline & B vs C & NB & & & $*$ & & & NB & & & $*$ & & & & & & & & \\
\hline \multicolumn{20}{|c|}{ 2. Physical Working Capacity - Relative [Peakload [Watt/Kg] } \\
\hline \multirow{3}{*}{ Groups } & $\begin{array}{c}\mathrm{A}(\mathrm{n}=13) \\
\Delta \%\end{array}$ & 1.8 & 0.58 & $\begin{array}{l}1.2 \\
3.1\end{array}$ & $\begin{array}{l}2.16 \\
20 \%\end{array}$ & 0.53 & $\begin{array}{l}1.4 \\
3.2\end{array}$ & $\begin{array}{c}2.0 \\
11.1 \%\end{array}$ & 0.48 & $\begin{array}{l}1.4 \\
2.8\end{array}$ & $\begin{array}{c}2.0 \\
11.1 \%\end{array}$ & 0.49 & $\begin{array}{l}1.4 \\
3.1\end{array}$ & $\begin{array}{c}* \\
0.01\end{array}$ & $\begin{array}{c}* \\
0.03\end{array}$ & $\begin{array}{c}* \\
0.01\end{array}$ & $\begin{array}{c}* \\
0.01\end{array}$ & $\begin{array}{c}* \\
0.01\end{array}$ & 0.83 \\
\hline & $\begin{array}{c}\mathrm{B}(\mathrm{n}=16) \\
\Delta \%\end{array}$ & 1.9 & 0.32 & $\begin{array}{l}1.3 \\
2.5\end{array}$ & $\begin{array}{c}2.24 \\
17.9 \%\end{array}$ & 0.30 & $\begin{array}{l}1.7 \\
2.8\end{array}$ & $\begin{array}{c}2.1 \\
10.5 \% \\
\end{array}$ & 0.30 & $\begin{array}{l}1.6 \\
2.8\end{array}$ & $\begin{array}{c}2.4 \\
26.3 \%\end{array}$ & 0.34 & $\begin{array}{l}1.7 \\
3.1\end{array}$ & $\begin{array}{c}* \\
0.01\end{array}$ & $\begin{array}{c}* \\
0.03 \\
0.03\end{array}$ & $\begin{array}{c}* \\
0.01\end{array}$ & $\begin{array}{c}* \\
0.01\end{array}$ & $\begin{array}{c}* \\
0.04 \\
\end{array}$ & $\begin{array}{c}* \\
0.01 \\
\end{array}$ \\
\hline & $\begin{array}{c}\mathrm{C}(\mathrm{n}=9) \\
\Delta \%\end{array}$ & 1.7 & 0.32 & $\begin{array}{l}1.3 \\
2.3\end{array}$ & $\begin{array}{c}1.8 \\
5.9 \%\end{array}$ & 0.35 & $\begin{array}{l}1.3 \\
2.3\end{array}$ & $\begin{array}{c}1.8 \\
5.9 \%\end{array}$ & 0.31 & $\begin{array}{l}1.3 \\
2.2\end{array}$ & $\begin{array}{c}1.8 \\
5.9 \%\end{array}$ & 0.25 & $\begin{array}{l}1.5 \\
2.2\end{array}$ & 0.47 & 0.14 & 0.92 & 0.65 & 0.49 & 0.53 \\
\hline \multirow{3}{*}{$\begin{array}{l}\text { Intergroup } \\
\text { Differences }\end{array}$} & A vs B & NB & & & NB & & & NB & & & NB & & & & & & & & \\
\hline & A vs $\mathrm{C}$ & NB & & & NB & & & NB & & & NB & & & & & & & & \\
\hline & B vs C & NB & & & $*$ & & & NB & & & $*$ & & & & & & & & \\
\hline \multicolumn{20}{|c|}{ 3. Body Mass (kg) } \\
\hline \multirow{3}{*}{ Groups } & $\begin{array}{c}\mathrm{A}(\mathrm{n}=13) \\
\Delta \%\end{array}$ & 84.7 & 9.7 & $\begin{array}{c}71.0 \\
104.5 \\
\end{array}$ & $\begin{array}{l}85.9 \\
1.4 \% \\
\end{array}$ & 9.0 & $\begin{array}{c}71.3 \\
105.0 \\
\end{array}$ & $\begin{array}{r}87.5 \\
3.3 \% \\
\end{array}$ & 9.8 & $\begin{array}{c}72.0 \\
105.5 \\
\end{array}$ & $\begin{array}{c}87.0 \\
2.7 \% \\
\end{array}$ & 9.5 & $\begin{array}{c}72.0 \\
103.5 \\
\end{array}$ & 0.19 & $\begin{array}{c}* \\
0.01\end{array}$ & $\begin{array}{c}* \\
0.01 \\
\end{array}$ & $\begin{array}{c}* \\
0.02 \\
\end{array}$ & 0.13 & 0.13 \\
\hline & $\begin{array}{c}\mathrm{B}(\mathrm{n}=16) \\
\Delta \%\end{array}$ & 85 & 11.0 & $\begin{array}{c}62.0 \\
102.5 \\
\end{array}$ & $\begin{array}{c}84.6 \\
-0.4 \% \\
\end{array}$ & 11.4 & $\begin{array}{c}61.0 \\
107.0 \\
\end{array}$ & $\begin{array}{c}85.1 \\
0.1 \% \\
\end{array}$ & 11.2 & $\begin{array}{c}62.0 \\
107.5 \\
\end{array}$ & $\begin{array}{c}84.2 \\
-0.9 \% \\
\end{array}$ & 10.5 & $\begin{array}{c}60.0 \\
102.5 \\
\end{array}$ & 0.61 & 0.8 & 0.27 & 0.31 & 0.51 & 0.51 \\
\hline & $\begin{array}{c}\mathrm{C}(\mathrm{n}=9) \\
\Delta \%\end{array}$ & 81.8 & 11.1 & $\begin{array}{l}65.0 \\
96.0\end{array}$ & $\begin{array}{l}82.8 \\
1.2 \% \\
\end{array}$ & 11.5 & $\begin{array}{l}66.5 \\
99.5 \\
\end{array}$ & $\begin{array}{l}83.9 \\
1.6 \% \\
\end{array}$ & 11.5 & $\begin{array}{c}67.5 \\
100.0 \\
\end{array}$ & $\begin{array}{c}83.5 \\
2.1 \% \\
\end{array}$ & 11.7 & $\begin{array}{c}67.5 \\
101.0 \\
\end{array}$ & 0.09 & $\begin{array}{c}* \\
0.04 \\
\end{array}$ & $\begin{array}{c}* \\
0.02 \\
\end{array}$ & 0.18 & 0.08 & 0.08 \\
\hline \multirow{3}{*}{$\begin{array}{l}\text { Intergroup } \\
\text { Differences }\end{array}$} & & NB & & & NB & & & NB & & & NB & & & & & & & & \\
\hline & & NB & & & NB & & & NB & & & NB & & & & & & & & \\
\hline & & NB & & & NB & & & NB & & & NB & & & & & & & & \\
\hline
\end{tabular}

A = Experimental Group A [Trained @ 3 Times/Week - Retrained @ 2/Week].

B = Experimental Group B [Trained @ 3 Times/Week - Retrained @ 4/Week].

$\mathrm{C}=$ Control Group: No Training or Retraining.

$*=\mathrm{p} \leq 0.05$.

$\Delta \%=$ Percentage Difference from Baseline Value 
Table 2: The Effect of Training Frequency on Some Selected Biochemical Health Parameters in the Training and Retraining of Sedentary Male Employees 1. Total Cholesterol Concentration (Mg/Dl) (TC)

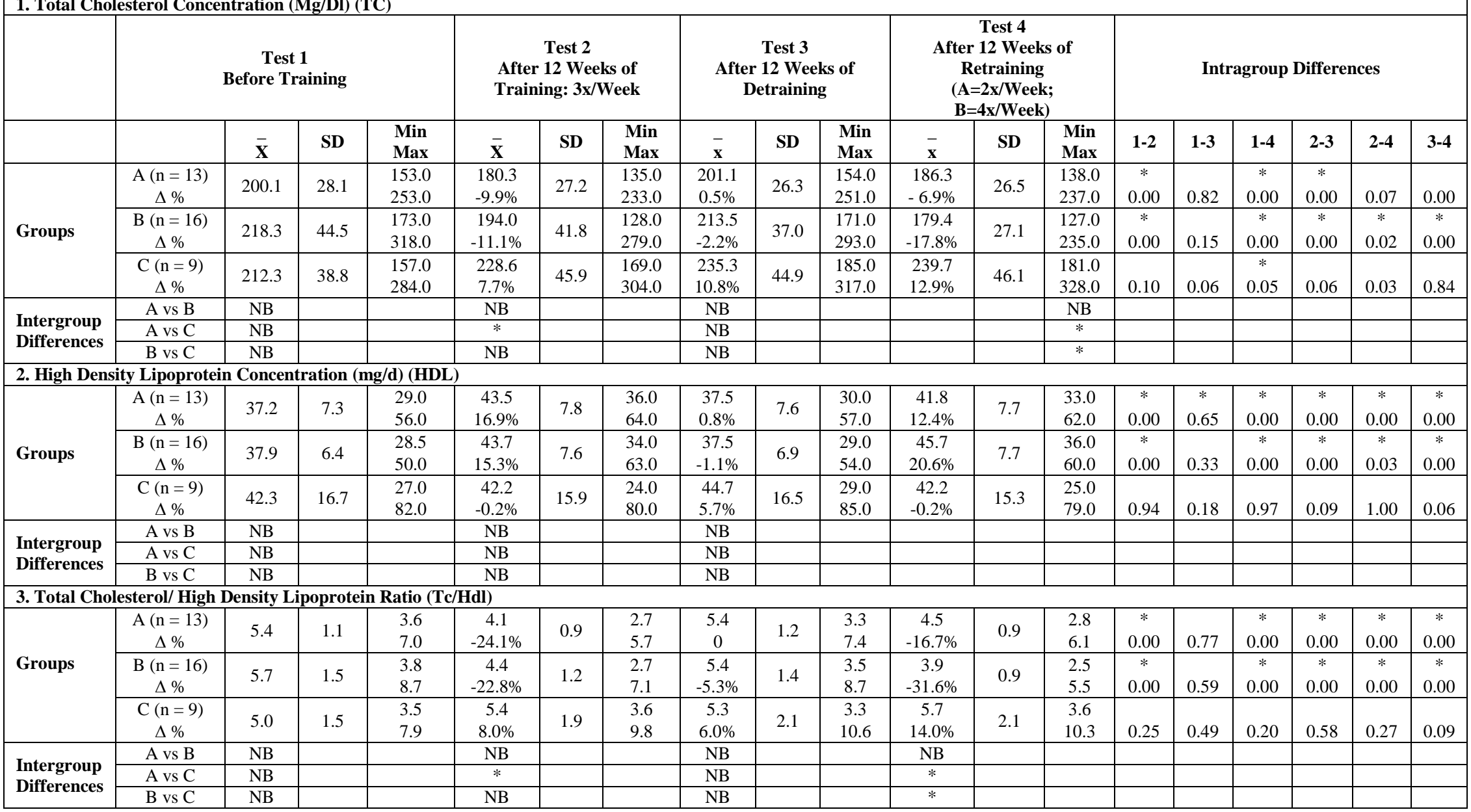


Table 2 cont.

\begin{tabular}{|c|c|c|c|c|c|c|c|c|c|c|c|c|c|c|c|c|c|c|c|}
\hline \multicolumn{20}{|c|}{ 4. Low Density Lipoprotein Concentration (LDL) (Mg/DI) } \\
\hline & \multicolumn{4}{|c|}{$\begin{array}{c}\text { Test } 1 \\
\text { Before Training }\end{array}$} & \multicolumn{3}{|c|}{$\begin{array}{c}\text { Test } 2 \\
\text { After 12 Weeks of } \\
\text { Training: 3x/Week }\end{array}$} & \multicolumn{3}{|c|}{$\begin{array}{c}\text { Test } 3 \\
\text { After } 12 \text { Weeks of } \\
\text { Detraining }\end{array}$} & \multicolumn{3}{|c|}{$\begin{array}{c}\text { Test } 4 \\
\text { After } 12 \text { Weeks of } \\
\text { Retraining } \\
(A=2 x / \text { Week; } \\
B=4 x / \text { Week })\end{array}$} & \multicolumn{6}{|c|}{ Intragroup Differences } \\
\hline & & $\overline{\mathbf{X}}$ & SD & $\begin{array}{l}\text { Min } \\
\text { Max }\end{array}$ & $\overline{\mathbf{X}}$ & SD & $\begin{array}{l}\text { Min } \\
\text { Max }\end{array}$ & $\overline{\mathbf{x}}$ & SD & $\begin{array}{l}\text { Min } \\
\text { Max }\end{array}$ & $\overline{\mathbf{x}}$ & SD & $\begin{array}{l}\text { Min } \\
\text { Max }\end{array}$ & $1-2$ & $1-3$ & $1-4$ & $2-3$ & $2-4$ & 3-4 \\
\hline \multirow{3}{*}{ Groups } & $\begin{array}{c}\mathrm{A}(\mathrm{n}=13) \\
\Delta \%\end{array}$ & 139.3 & 33.4 & $\begin{array}{c}85.0 \\
192.0\end{array}$ & $\begin{array}{c}117.1 \\
-15.9 \%\end{array}$ & 33.2 & $\begin{array}{c}63.0 \\
167.0\end{array}$ & $\begin{array}{l}134.5 \\
-3.4 \%\end{array}$ & 33.3 & $\begin{array}{c}78.0 \\
187.0\end{array}$ & $\begin{array}{c}117.6 \\
-15.6 \%\end{array}$ & 31.8 & $\begin{array}{c}60.0 \\
165.0\end{array}$ & $\begin{array}{c}* \\
0.00\end{array}$ & $\begin{array}{c}* \\
0.00\end{array}$ & $\begin{array}{c}* \\
0.00\end{array}$ & $\begin{array}{c}* \\
0.00\end{array}$ & 0.85 & $\begin{array}{c}* \\
0.00\end{array}$ \\
\hline & $\begin{array}{c}\mathrm{B}(\mathrm{n}=16) \\
\Delta \%\end{array}$ & 153.2 & 43.3 & $\begin{array}{c}90.0 \\
239.0\end{array}$ & $\begin{array}{c}132.4 \\
-13.6 \%\end{array}$ & 406 & $\begin{array}{c}71.0 \\
214.0\end{array}$ & $\begin{array}{r}151.9 \\
-0.8 \%\end{array}$ & 40.0 & $\begin{array}{c}86.0 \\
233.0\end{array}$ & $\begin{array}{c}122.3 \\
-20.2 \%\end{array}$ & 30.6 & $\begin{array}{c}74.0 \\
193.0\end{array}$ & $\begin{array}{c}* \\
0.00\end{array}$ & 0.43 & $\begin{array}{c}* \\
0.00\end{array}$ & $\begin{array}{c}* \\
0.00\end{array}$ & $\begin{array}{c}* \\
0.01\end{array}$ & $\begin{array}{c}* \\
0.00\end{array}$ \\
\hline & $\begin{array}{c}\mathrm{C}(\mathrm{n}=9) \\
\Delta \%\end{array}$ & 137.8 & 46.3 & $\begin{array}{c}68.0 \\
206.0\end{array}$ & $\begin{array}{l}145.6 \\
5.7 \%\end{array}$ & 48.2 & $\begin{array}{c}66.0 \\
210.0\end{array}$ & $\begin{array}{l}146.6 \\
6.4 \%\end{array}$ & 45.5 & $\begin{array}{c}73.0 \\
217.0\end{array}$ & $\begin{array}{l}148.8 \\
8.0 \%\end{array}$ & 45.5 & $\begin{array}{c}79.0 \\
226.0\end{array}$ & 0.07 & $\begin{array}{c}* \\
0.02\end{array}$ & $\begin{array}{c}* \\
0.01\end{array}$ & 0.67 & 0.36 & 0.15 \\
\hline \multirow{3}{*}{$\begin{array}{l}\text { Intergroup } \\
\text { Differences }\end{array}$} & A vs B & & & NB & & & NB & & & NB & & & NB & & & & & & \\
\hline & A vs C & & & NB & & & NB & & & NB & & & NB & & & & & & \\
\hline & $\mathrm{B}$ vs $\mathrm{C}$ & & & NB & & & NB & & & NB & & & NB & & & & & & \\
\hline \multicolumn{20}{|c|}{ 5. Triglyceride $(\mathrm{mg} / \mathrm{dl})$} \\
\hline \multirow{3}{*}{ Groups } & $\begin{array}{c}\mathrm{A}(\mathrm{n}=13) \\
\Delta \%\end{array}$ & 157.5 & 54.6 & $\begin{array}{c}84.0 \\
242.0\end{array}$ & $\begin{array}{c}124.1 \\
-21.2 \%\end{array}$ & 47.4 & $\begin{array}{c}71.0 \\
202.0\end{array}$ & $\begin{array}{l}157.9 \\
0.3 \%\end{array}$ & 61.3 & $\begin{array}{c}00.0 \\
231.0\end{array}$ & $\begin{array}{c}126.5 \\
-19.7 \% \\
\end{array}$ & 60.1 & $\begin{array}{l}00.00 \\
222.0\end{array}$ & $\begin{array}{c}* \\
0.00\end{array}$ & 0.31 & 0.11 & 0.46 & 0.89 & $\begin{array}{c}* \\
0.00\end{array}$ \\
\hline & $\begin{array}{c}\mathrm{B}(\mathrm{n}=16) \\
\Delta \%\end{array}$ & 148.2 & 48.1 & $\begin{array}{c}72.0 \\
204.0\end{array}$ & $\begin{array}{c}119.6 \\
-19.3 \%\end{array}$ & 32.9 & $\begin{array}{c}72.0 \\
165.0\end{array}$ & $\begin{array}{l}148.6 \\
0.3 \%\end{array}$ & 39.6 & $\begin{array}{r}74.0 \\
201.0\end{array}$ & $\begin{array}{c}112.6 \\
-24.0 \%\end{array}$ & 31.4 & $\begin{array}{c}71.0 \\
157.0\end{array}$ & $\begin{array}{c}* \\
0.00\end{array}$ & 0.89 & $\begin{array}{c}* \\
* \\
0.00\end{array}$ & $\begin{array}{c}* \\
* \\
0.00\end{array}$ & $\begin{array}{c}* \\
0.00\end{array}$ & $\begin{array}{c}* \\
0.00\end{array}$ \\
\hline & $\begin{array}{c}\mathrm{C}(\mathrm{n}=9) \\
\Delta \%\end{array}$ & 132.0 & 75.5 & $\begin{array}{c}60.0 \\
261.0\end{array}$ & $\begin{array}{c}145.3 \\
10.1 \% \\
\end{array}$ & 95.8 & $\begin{array}{c}61.0 \\
327.0\end{array}$ & $\begin{array}{l}139.3 \\
5.5 \%\end{array}$ & 85.5 & $\begin{array}{c}60.0 \\
277.0 \\
\end{array}$ & $\begin{array}{l}139.6 \\
5.8 \% \\
\end{array}$ & 86.1 & $\begin{array}{c}59.0 \\
282.0\end{array}$ & 0.12 & 0.17 & 0.15 & 0.32 & 0.30 & 0.86 \\
\hline \multirow{3}{*}{$\begin{array}{l}\text { Intergroup } \\
\text { Differences }\end{array}$} & $\mathrm{A}$ vs B & NB & & & NB & & & NB & & & NB & & & & & & & & \\
\hline & A vs C & NB & & & NB & & & NB & & & NB & & & & & & & & \\
\hline & B vs C & NB & & & NB & & & NB & & & NB & & & & & & & & \\
\hline
\end{tabular}


As previously indicated, the initial training phase of the two experimental groups (A \& B) took place at a frequency of three times per week. According to the ACSM (2010), this frequency is sufficient to provoke training adaptations, provided that the programme also complies with the indicated levels of the other training principles (ACSM, 2010).

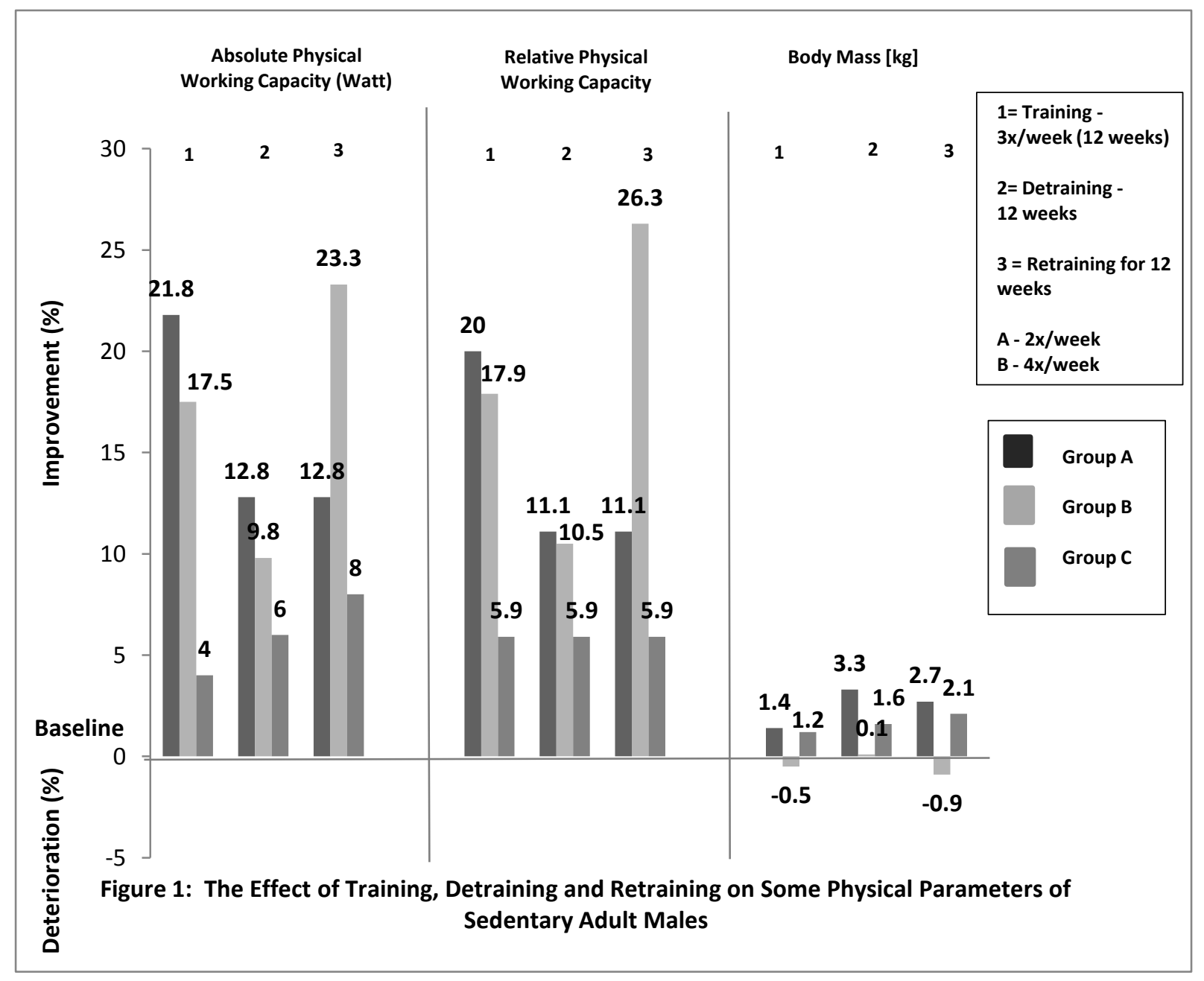

From Table 1 and Figure 1, it is clear that both training groups (A \& B) showed a statistical significant improvement in the physical working capacity (PWC - Absolute \& Relative) after the initial training phase. After detraining, the PWC deteriorated but still remains statistically significantly better than during the baseline assessment (12.8\% \& 9.8\% for the PWC-absolute and $11.1 \%$ \& $10.5 \%$ for the PWC- relative values, respectively). After retraining for another 12 weeks, Group B, who trained for four times per week, once again increased their physical working capacity (absolute \& relative) while Group A, who trained at a frequency of two times per week, remained the same. Inconsistent changes occurred in the body mass of the various groups. In the two exercising groups (A \& B), Group A showed an increase after the initial training period, while Group B experienced a decrease in body mass. After detraining, Group A experienced a further increase in body mass, with Group B only showing a slight increase compared to the baseline value. With retraining, Group A showed 2.7\% increase in body mass while Group B showed a slight loss $(-0.9 \%)$. The control group (C) indicated a gradual increase in body mass with each assessment. 


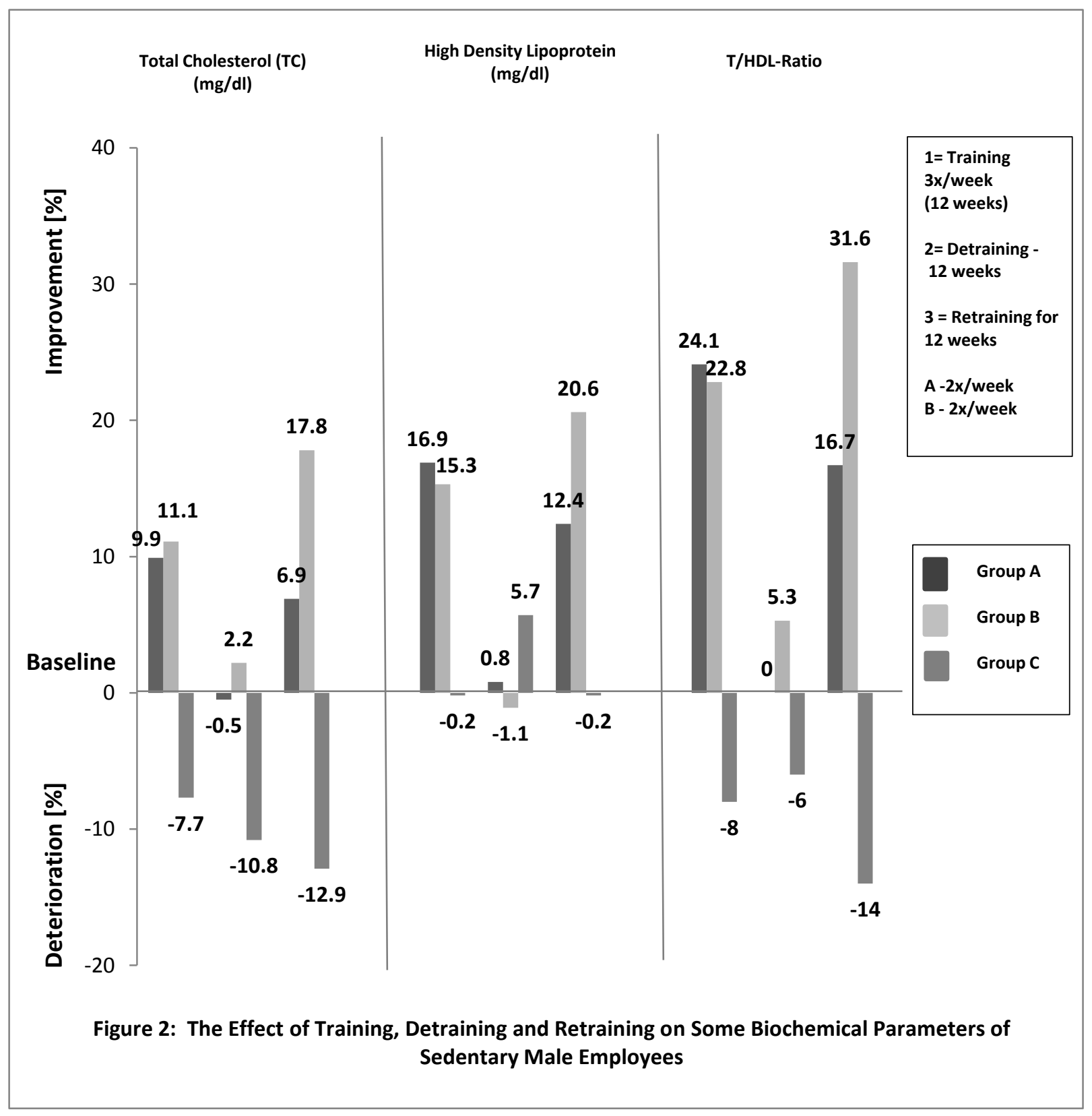

As far as the lipid profile response is concerned, the exercising groups (A \& B) showed a decrease in the total cholesterol (TC), low-density lipoprotein (LDC), TC/HDL-ratio and triglyceride (TG) concentration after the conditioning phase. This decrease represents an "improvement" in the risk factors (Figure 2 and Figure 2 Continue). The high-density lipoprotein (HDL), on the other hand, showed an increase after the initial conditioning, also representing an improvement in the lipid profile, as it is considered "good" cholesterol, while first mentioned parameters are all associated with higher incidence of cardiovascular disease (Nieman, 1998; Visich \& Fletcher, 2009; ACSM, 2010). These changes were all statistically significant. After the detraining phase of 12 weeks (T3), the activity groups (A \& B) almost returned to the pre-exercising level in all the parameters.

After retraining, Groups A and B once more depicted improvement in all parameters, but Group B, who trained at a higher frequency (four times per week), showed the largest improvement, especially in the TC, HDL and TC/HDL ratio. 


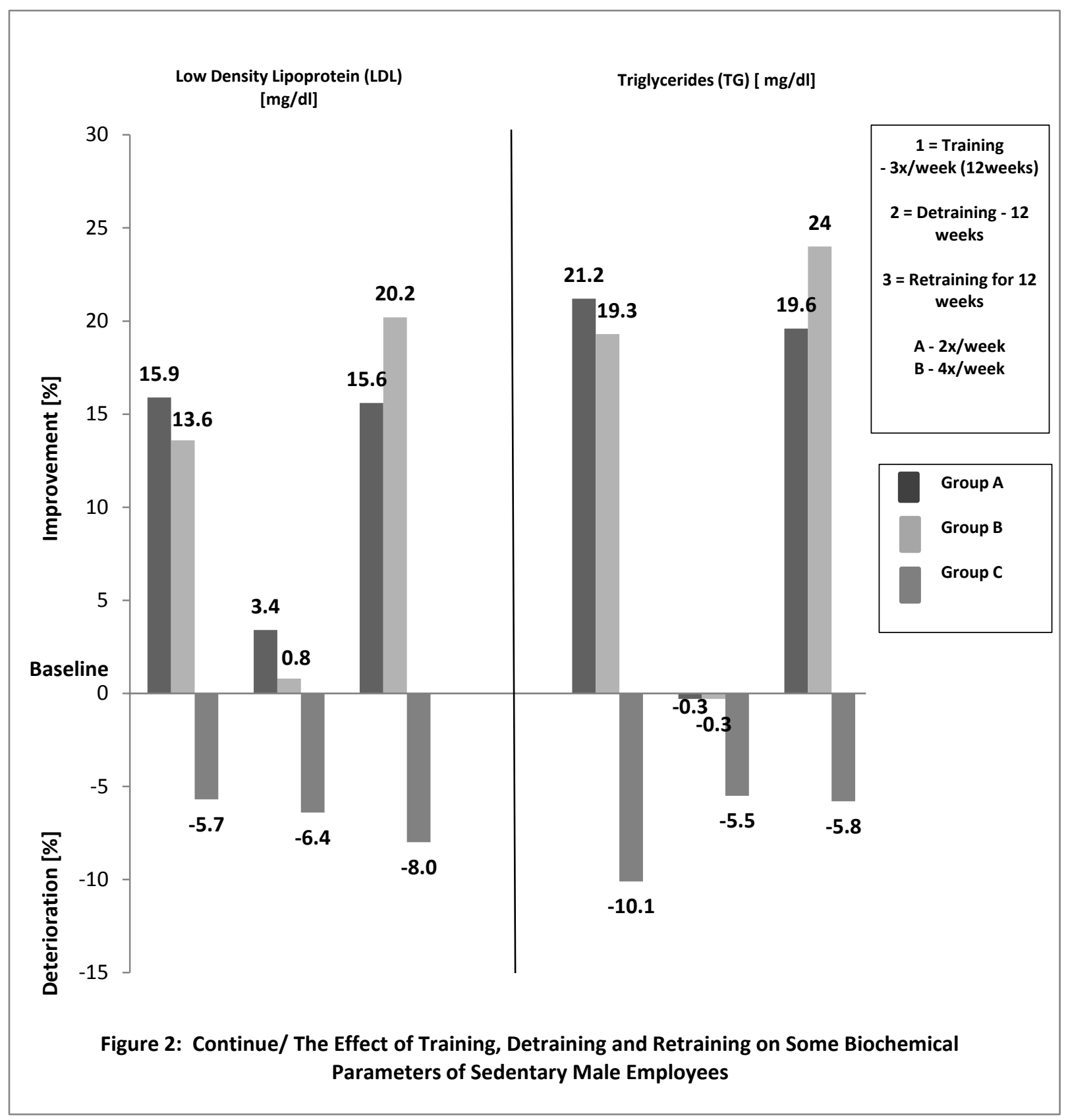

Thus, an improvement of almost $50 \%$ was indicated when compared to Group A, who retrained at a frequency of two times per week. As far as the control group is concerned, all parameters showed a deterioration compared to the baseline values, with the HDL indicating the smallest changes (Figure 2 and Figure 2 Continue).

\section{DISCUSSION}

By increasing the physical working capacity and health status of employees, a more effective workforce, leading to possible improvement of productivity, lower presenteeism, absenteeism and a decrease in health care cost, may be established (Burton, McCalister, Chen \& Edington, 2005; Labuschagne, 2006). A low physical working capacity, or aerobic fitness, is not only identified as one of the four primary risk factors for coronary heart disease (CHD) (Nieman, 1998; ACSM, 2010) but is also associated with the other primary risk factors - hypertension, 
hypercholesterolemia and smoking (ACSM, 2010). Apart from the salutogenic effect of physical training on the CHD risk factors, it is also demonstrated that an improved cardiorespiratory fitness can cause a protective outcome in CHD mortality, even in the presence of primary risk factors - high cholesterol, hypertension and smoking (Barlow, Brill, Blair \& Kohl, 1990).

From Table 1, it is clear that after the initial training phase of 12 weeks, a significant increase occurred in the PWC (Absolute \& Relative) of the exercising groups (A \& B). During detraining, both groups lost about 50\% of their initial gains over the 12 weeks of inactivity. With retraining at a frequency of two times per week (Group A), the PWC was maintained. This is in line with previous research indicating that with retraining at a frequency of two times per week, the physical capacity will be maintained, while a frequency of one time per week will lead to a slight deterioration (Fox \& Mathews, 1981; Opperman \& Strydom, 2012). A frequency of four times per week or more will increase the salutogenic effect as it has already been established that the outcome is exercise dose dependant (ACSM, 2010).

From the results of this study, it is clear that a training frequency of three times per week provokes significant physiological adaptations leading to improved aerobic capacity (ACSM, 2010). In the retraining phase, a frequency of two times per week was adequate to maintain the aerobic capacity of Group A, while Group B, who trained four times per week, the aerobic capacity exceeded the initial training phase of three times per week. The contribution, in this regard, for an employee wellness program is that a short interruption of the training programme will not cause significant back slide of gained aerobic improvement, especially when the individual may be motivated to participate in some form of physical activity for at least two times per week. It is interesting to note that in the case of highly trained athletes, a rapid drop in aerobic endurance occurred after the first three weeks of training cessation, while for people with low to moderate levels of aerobic fitness, little change occurred in stamina during the first three weeks, but quickly reversed to the pre-exercise level after further weeks of physical inactivity (http://www.tinajuanfitness.info/articles/113004.htm). According to research, the initial rapid loss of aerobic capacity occurs parallel with the loss of blood plasma volume (Lee, Moore, Everette, Stenger \& Platts, 2010).

In the case of strength training, the opposite response takes place (Taaffe \& Marcus, 2000). It was indicated that a rapid decline occurred in the muscle strength over the first two weeks of detraining but then basically remained the same for the next six weeks, with some further decrease in the following weeks (Taaffe \& Marcus, 2000).

As far as the lipid parameters are concerned, it seems that the mean values of TC, LDL and TC/HDL ratio of all the groups (A, B \& C), as well as the HDL for Groups A and B during the baseline assessment, can be classified in the "borderline high" category. Only Group A can be regarded in this risk group as far as the TG concentration is concerned. This profile places the individuals into a higher risk for developing coronary heart disease (Biggerstaff \& Wooten, 2009) which may increase health care cost (Chenoweth, 2011). According to KolbeAlexander et al. (2008), 10.6\% - 32.5\% (mean 18.6\%) of the employees in various corporate environments in South Africa showed elevated TC (> $200 \mathrm{mg} . \mathrm{dl}$ ). Other health risks, as indicated by Kolbe-Alexander et al. (2008), were increased BMI (48.6\%), systolic blood pressure (13.0\%), diastolic blood pressure (12.2\%), physical inactivity (69\%) and smoking (19.9\%). According to Dreyer (1996), 29\% of the South African male executives showed three of the primary risk factors which can be associated with coronary heart disease, while $43 \%$ and $25 \%$ showed one or two risk factors, respectively. These figures are in line with the research, indicating that currently $37 \%$ of the deaths in South Africa can be attributed to non-communicable disease (Kalas et al., 2012). The high prevalence of health risk factors in the South African corporate environment lead to the implementation of employee wellness programmes in South Africa during the mid 1980's (Kalas et al., 2012). As previously indicated, training at a frequency of three times per week leads to a significant reduction in TC, TC/HDL ratio, LDL and TG, with an increase in HDL. These improvements in a risky lipid profile may lead to a decrease in cardiovascular morbidity and mortality. In this regard, Nieman (1998) indicates that for every $1 \%$ reduction in TC, the occurrence of coronary heart disease is reduced by $2-3 \%$, which may also reduce the health care costs.

With detraining, it seems as if all the positive gains in the lipid profile are nearly completely lost. With retraining of both groups, an improvement in the various lipid profiles was indicated, with Group B exceeding the improvement in Group A in all the variables. This supports the notion of the dose-dependent relationship between 
physical training and lipid parameters as Group B retrained at a frequency of four times per week compared to the two times per week of Group A (Nieman, 1998). The mechanisms responsible for the "improvement" of the lipid profile resulting from an exercise intervention is probably related to the increased activity in lipoprotein lipase (LPL), cholesterol-ester transfer protein activity (CETPA) and lecithin cholesterol acyltransferase activity (LCAT) (Biggerstaff \& Wooten, 2009).

Some literature suggests that when other changes, which may occur with exercise training, are ignored change in body mass and diet, training may only provoke an improvement in HDL (increase) and TG (decrease) while TC and LDL remain fairly constant (Nieman, 1998; Mestek, 2009). In this study, the response of body mass to training and retraining was inconsistent. Group A experienced an increase in body mass (1.4\%) while Group B showed a slight decrease $(-0.4 \%)$ during the initial training phase. With retraining, Group A showed a $2.7 \%$ increase on baseline data, while Group B had a $0.9 \%$ decrease. These differences could be related to the higher frequency of training of Group B. The control group showed a progressive increase in body mass over the period of time.

\section{LIMITATIONS}

A limitation of this study was the small number of participants who finally completed the study. The research also represents the situation at one tertiary institution and should not be generalized.

\section{CONCLUSION}

It is clear that an intervention regime of physical exercise can improve some of the primary risk factors associated with coronary heart disease - physical inactivity, low HDL, elevated TC, LDL, TG and TC/HDL-ratio. This improvement is not associated with a consistent response in body mass of the experimental groups, especially during the initial training phase where both groups trained at the same intensity. During the retraining phase, Group $\mathrm{B}$ trained at a higher frequency (four times per week) and a decrease in body mass could be expected. It is also clear that health gains starts decreasing with detraining, but it seems that the decrease in physical working capacity is less over the detraining phase of 12 weeks, than in the case of the biochemical parameters.

This enforces the notion that when embarking on an intervention programme, aiming at the improvement of cardiovascular health, participants should try to keep up their physical activity habits in order to maintain the salutogenic adaptation. A frequency of two times per week could maintain some of the gained benefits of training. The desired frequency, however, is to train at a frequency of three or more times per week in order to obtain optimum results (ACSM, 2010).

\section{AUTHOR INFORMATION}

Marlene Opperman is a senior lecturer at the University of the Free State, South Africa. She received her BA, Hons BA, MA and Ph.D from the Potchefstroom University (the MA with distinction). Her research is mainly in the field health sciences. She was a promoter of a doctoral thesis and several master dissertations as well as acted as external examiner for many Honnors, Masters and Doctorate students at various national universities. Dr. Opperman delivered several presentations on scientific conferences in South Africa. She also regularly acts as guest lecturer, evaluator and examiner for the Sport Medicine program (M.Phil) at her University. E-mail: oppermm@ufs.ac.za

Gert L. Strydom is an emeritus-professor (Potchefstroom Campus) and an extraordinary professor (Mafikeng Campus) of the North-West University in South Africa; he is also visiting professor at the University of Venda. He received his BA, Hons BA, MA and DPhil from the Potchefstroom University, all three postgraduate degrees with distinction. He is also the holder of an honorary doctorate from the North-West University for his dedicated work in the academic environment. Prof Strydom is doing research over a broad spectrum of disciplines with the main research field health science; the health discipline Biokinetics, registered as the profession biokinetics/clinical exercise physiologist with the Health Professional Council of South Africa, originated from his research and creative leadership. He is affectionately called the "South African Father of Biokinetics." He published extensively in peer reviewed and accredited journals, locally and internationally, and acted as the sole and co-author for many books. He was a promoter for many doctoral theses and masters dissertations as well as other research projects and is still 
very active in this domain. Prof Strydom delivered many presentations on scientific conferences, in South Africa and abroad. E-mail: mbwgls@puknet.puk.ac.za (Corresponding author)

\section{REFERENCES}

1. American College of Sports Medicine. (ACSM) (2010). Guidelines for exercise testing and prescription ( $8^{\text {th }}$ ed.). Philadelphia: Lippincott Williams \& Wilkens.

2. Barlow, C. E., Brill, P. A., Blair, S. V., \& Kohl, H. W. (1990). Practical advice on fitness and mortality. A new approach to exercise presentation. American Journal of Health Promotion, 4(10), 391-393.

3. Biggerstaff, K. D., \& Wooten, J. S. (2009). Hyperlipidemia and dyslipidemia In. J. K. Ehrman, P. M. Gordon, P. S Visich, \& S. T. Keteyian (Eds.), Clinical exercise physiology (pp. 247-264). Champaign Ill. Human Kinetics.

4. Booth, F. W., Gordon, S. E., Carlson, C .J., \& Hamilton, M. T. (2000). Waging war on modern chronic diseases, primary prevention through exercise biology. Journal of Applied Physiology, 88, 774-787.

5. Burstein, M., Scholnick, H. R., \& Morfin, R. (1970). Rapid methods for the isolation of lipoprotein from human serum by precipitation with polyanious. Journal of Lipid Research, 11(6), 583-595.

6. Burton, W. N., McCalister, K. T., Chen, C.-Y., \& Edington, D. W. (2005). The associaton of health status, worksite fitness centre participation and two measures of productivity. Journal of Environmental and Occupational Medicine, 47(4), 343-351

7. Chenoweth, D. H. (2011). Worksite health promotion $\left(3^{\text {rd }}\right.$ Ed). Champaign Ill. Human Kinetics.

8. Dreyer, L. I. (1996). Die effek van inoefening op enkele koronêre risikofaktore en hulle onderlinge verwantskap by Suid-Afrikaanse bestuurlui. (Ph.D - Thesis), PU for CHE, Potchefstroom.

9. Edington, D. W. (2006). Who are the intended beneficiaries (targets) of employee health promotion and wellness programs? NC Med J, 67(6), 425-427.

10. Cole, T. G, Klotzsch, S. G., \& Namara, M. C. (1997). Measurement of triglyceride concentration. In N. Rifai, G.R. Warnick \& M.H. Domiminiczak, (Eds.). Handbook of lipoprotein testing (pp. 115-126). A AAC Press, Washington.

11. Edington, D. W. (2001). Emerging research: A view from one research center. The Science of Health Promotion, 15(5), 341-349.

12. Fox, E. L., \& Mathews, D. K. (1981). The physiological basis of physical education and athletics. New York. Saunders College Publishing.

13. Friedewald, W. T., Levy, R. I., \& Friederickson, D. S. (1972). Estimation of the concentration of lowdensity lipoprotein cholesterol in plasma, without use of preparative ultra-centrifuge, Clinical Chemistry, $18,449-502$.

14. Grace, J. M., Wilders, C. J., Strydom, G. L., \& Ellis, S. (2012). A comparison of the effect of two intervention regimes on coronary prone executives in the South Africa colliery industry. Occupational Health, Southern Africa, 18(1), 15-21.

15. Http://www.tinajuanfitness.info/articles/113004.htm. What happens to your body when you stop exercising. Retrieved 23 April 2012.

16. IFPMA, (2011), Cost of Non- Communicable Diseases. Retrieved 4 March 2013 from http://www.lfpma.org/fileadmin/content/Global\%20 Health NCDs

17. Kalas, T., Kirsten, W., Strydom, G., \& Wilders, C. (2012). Health promotion in South Africa In W. Kirsten \& R. C. Karch (Eds.), Global perspectives in workplace health promotion. London, Jones \& Bartlett Learning International.

18. Kolbe-Alexander, T. L., Buckmaster, C., Nossel, C., Dreyer, L., Bull, F., Noakes, T. D., \& Lambert, E. V. (2008). Chronic disease risk factors, healthy days and medical claims in South African employees presenting for health screening. BMC Public Health, 8, 228, Article available from: http://www.biomedicalcentral.com/1471-2458/8/228

19. Labuschagne, R. (2006). Fisieke aktiwiteit en enkele gesondheidaspekte by werknemers aan in finansiële instelling. (MA. Dissertation). Potchefstroom: North-West University.

20. Lambert, E. V., \& Kolbe-Alexander, T. (2006). Physical activity and chronic diseases of lifestyle in South Africa. In K. Steyn, J. Fourie \& J.Temple (Eds.), Chronic diseases of lifestyle in South Africa: 1995 - 2005 (pp. 23-32). Medical Research Council Technical Report. 
21. Lee, S. M. C, Moore, A. D., Everette, M. E., Stenger, M. B., \& Platts, S. H. (2010). Aerobic exercise deconditioning and counter measures during bedrest. Aviation Space and Environmental Medicine, 81(1), 52-63.

22. Mc Ardle, W. D., Katch, F. I., \& Katch, V. L. (1996) Exercise physiology (4 ${ }^{\text {th }}$ ed.). London, Williams \& Wilkens.

23. Mestek, M. L. (2009). Physical activity, blood lipids and lipoproteins. American Journal of Lifestyle Medicine, 3(4), 279-283.

24. Musich, S., McDonald, T. Hirschland, D., \& Edington, D.W. (2003). Examination of risk status among active employees in a comprehensive worksite health promotion program. Journal of Occupational and Environmental Medicine, 45(4), 393-399.

25. Nieman, D. C. (1998). The exercise health connection Champaign, Ill. Human Kinetics.

26. Oberg, E. (2007). Physical activity prescription: our best medicine. Integrative Medicine: A Clinician's Journal, 6(5), 18-22.

27. Opperman, M. C. \& Strydom, G. L. (2012). The effect of training frequency on selected physical and hemodynamic parameters in the training and retraining of sedentary adult males. African Journal for Health, Physical Education, Recreation and Dance, 18(4:2), 1007-1020.

28. Richmond, W. (1973). Preparation and properties of a cholesterol oxidase from Nocardia sp and its application to the enzymatic assay of total cholesterol in serum. Clinical Chem, 19, 1350-1356.

29. Stokes, G. C., Henly, N. S., \& Herget, C. (2006). Creating a culture of wellness in workplaces. NC Med J, 67(6), 445-448.

30. Taaffe, D. R., \& Marcus, R. (2000). Musculoskeletal health and older adults. Journal of Rehabilitation Research \& Development, 37(2), 245-254.

31. Thomas, J. R., Nelson, J. K., \& Silverman, S. J. (2011). Research methods in physical activity (6 ${ }^{\text {th }}$ ed.). Champaign, Ill. Human Kinetics.

32. Visich, P. S., \& Fletcher, E. (2009). Myocordial infarction. In, J. K. Ehrman, P. M. Gordon, P. S. Visich \& S. J. Keteyian. (Eds.), Clinical exercise physiology. Champaign, Ill. Human Kinetics.

33. World Economic Forum. (2009). Global Risks Landscape Assessment Report. Cologny/Geneva, Switzerland.

34. World Economic Forum. (2013). The workplace wellness alliance. Making the right investment: Employee health and the power of metrics. Cologny/Geneva, Switzerland. 